\title{
Commentary - The late preterm infant: Vulnerable cerebral cortex and large burden of disability
}

\author{
J.J. Volpe $\mathrm{a}^{\mathrm{a}, \mathrm{b}, *}$ \\ ${ }^{a}$ Department of Neurology, Harvard Medical School, Boston, MA, USA \\ ${ }^{\mathrm{b}}$ Department of Pediatric Newborn Medicine, Harvard Medical School, Boston, MA, USA
}

Received 22 May 2021

Accepted 6 June 2021

Keywords: Cerebral cortical dysmaturation, late preterm, neurodevelopmental disability

\section{Introduction}

The principal focus of this Commentary is the late preterm (LPT) infant and, especially, the generally underappreciated, enormous burden of neurodevelopmental disability (NDD) observed subsequently in these infants. LPT infants, defined by birth at 34-36 completed weeks of gestation, i.e., at 34-36 $6 / 7$ weeks, comprise by far the largest proportion of preterm infants (37 weeks' gestation or less). In 2019 in the US LPT infants accounted for $7.46 \%$ of the 3,745,540 live births in the US, i.e., approximately 280,000 such infants and approximately $75 \%$ of all preterm births [1]. The rate of LPT births has increased steadily over the past 5 years. LPT infants have increased rates of perinatal morbidities as well as subsequent NDD (see later). However, importantly, a major proportion of the NDD occurs in the absence of recognized perinatal problems [2]. The absolute number of LPT infants with subsequent NDD yearly is approximately 3,500 [3]. The enormous emotional and financial costs for families and

*Address for correspondence: J.J. Volpe, E-mail: Joseph.volpe @ childrens.harvard.edu. society attributable to such disability are extensive [3]. As will be discussed, although the neuroanatomic basis of this large burden of disability remains to be defined clearly, disturbance of cerebral cortical development appears central. This notion is supported by clinical and imaging data. Recent studies based on advanced MRI also have elucidated particularly rapid developmental events involving cerebral cortex in normal human brain at the particular time period of LPT birth (see later). These recent findings suggest an especial vulnerability of the developing cerebral cortex of the LPT infant and suggest opportunities for interventions to reduce the large burden of disability.

\section{Neurodevelopmental disability in LPT infants}

A large series of recent studies have elucidated the specific deficits encompassed by the subsequent NDD in LPT infants $[2,4-8,9]$. Although the specific deficits are not marked, they are appreciable enough to lead to impaired academic performance. Cognitive areas affected include executive function, 
literacy skills, verbal memory, attention, processing speed and overall school performance. Behavioral problems, autism and later psychiatric disturbances have been noted. Notably, in one expansive review that excluded LPT infants with "postnatal morbidity" ( $20 \%$ of the total), the deficits still could be defined clearly [10]. The array of deficits are most consistent with disturbances of various areas of cerebral cortex and their interconnections.

Although motor deficits are generally not emphasized, approximately $0.6-1.0 \%$ of LPT infants are reported to develop "cerebral palsy". Thus, approximately 2,000 cases per year of such significant motor disturbance may be attributable to LPT infants in the USA. These infants are principally those that experience such perinatal morbidities as low Apgar scores, need for resuscitation at birth, neonatal sepsis, intracranial hemorrhage or hypoglycemia $[11,12]$. (Note that the large group of infants with cognitive and related deficits described above did not experience serious neonatal morbidities.)

\section{Brain abnormality in LPT infants}

The general approach to definition of brain abnormality underlying neurological deficits in LPT infants, as at older ages, relies on postmortem neuropathological studies and imaging assessments on living infants. Both such studies in LPT infants have provided valuable and complementary information.

The most detailed neuropathological evaluations suggested that white matter injury (WMI) is common in LPT infants. Thus, Haynes et al. [13] studied 16 LPT infants and identified periventricular leukomalacia (PVL) in 63\%. The cellular features were similar to those observed in early PT infants [14], not unexpectedly because the principal cellular target in PVL, the pre-myelinating oligodendrocyte, comprises the majority of the oligodendroglial lineage during the LPT period [15]. Neuronal and axonal injury occurred in a minority of the cases and was confined to the thalamus. Importantly, because conventional neuropathology cannot detect the hallmarks of neuronal maturation, e.g., dendritic development, impaired neuronal development could not be assessed. Importantly, also, these deceased infants had experienced serious neonatal morbidities, e.g., respiratory distress syndrome, necrotizing enterocolitis, sepsis, unlike the large proportion of LPT infants described earlier with cognitive deficits but no major neonatal morbidities. It is, however, likely that the
WMI underlies the cerebral palsy described earlier in LPT infants with neonatal morbidities.

Studies of living LPT infants with MRI methods appear to provide better insights into the neuroanatomic substrate of the cognitive deficits described earlier. Thus, although there is some evidence for impaired microstructural development in cerebral white matter $[16,17]$, the preponderance of evidence indicates more important disturbances of cerebral cortical development. Studies utilizing various MRI methods and supporting this conclusion have shown in LPT infants, smaller cerebral cortical volumes, impaired cortical gyral development, and altered cerebral connectivity $[16,18-26]$. The findings have been observed as early as term-equivalent age and as late as pre-adolescence. Although LPT infants exhibit increased risk for need for resuscitation, respiratory distress, intermittent hypoxemia, hypothermia, hypoglycemia, hyperbilirubinemia and feeding difficulties $[3,26,27]$, no consistent relationships with the disturbed cerebral cortical development have been delineated. The possibilities that these factors operating in various combinations or that ex-utero life in the prematurely born infant "reprograms" or otherwise alters cerebral cortical development are worthy of consideration. The latter notion is supported by outcome studies in which such neonatal morbidities were said to be absent despite impaired neurodevelopment. A particular vulnerability of cerebral cortex likely relates to the rapidity of its development in the late preterm period, as discussed next.

\section{Cerebral cortical development in the LPT infant}

A major clue to the basis of the vulnerability of cerebral cortex in the LPT infant relates to the extraordinarily rapid and complex development occurring in the LPT period. This rapidity and complexity render the cerebral cortex vulnerable to disturbance by exogenous and endogenous factors related to premature extra-uterine life (see earlier). This development has been demonstrated both in anatomic and imaging studies.

Anatomic data show that overall the brain at 34 weeks still must gain fully $35 \%$ of its overall weight observed at term [13]. At the microscopic level, the cerebral cortex undergoes dramatic changes in the third trimester, with the most striking changes occurring during the last eight weeks [28]. Most prominent are the elaboration of dendritic and axonal 
ramifications, onset of synaptogenesis, a marked increase in cortical surface area and rapid gyral development [14]. The latter is especially prominent during the LPT period.

MRI studies with advanced techniques have described imaging correlates of this rapid cortical development in utero $[29,30]$. Thus, MRI of healthy fetuses has shown marked increases in cerebral volume in the third trimester, with volumes higher in fetuses versus comparably aged premature infants without observable parenchymal injury. A particularly large fetal MRI series $(n=166)$ showed a 21 -fold increase in cerebral cortical gray matter in the second half of gestation, and importantly for this discussion, the rate of increase from 30 weeks of gestation was more than twice that prior to 30 weeks. During the LPT time period cerebral cortical gray matter became the primary driver of the overall increase in cerebral volume [30].

Not unexpectedly, MRI studies of living premature infants have identified the period of 30-40 weeks of gestation as the time of most rapid increase in cerebral cortical volume [31-35]. Diffusion-based measurements of cerebral cortex are consistent with the role of cortical dendritic growth as the principal driver of the increases in cortical gray matter [34]. Importantly, the maturational changes become much less prominent after 37 weeks' gestation, the end of the LPT period.

Perhaps the most decisive demonstration of the importance of the LPT period regarding cerebral cortical development is provided by a recent MRI study utilizing serial scans of the same infants (unlike the longitudinal and cross-sectional studies of previous reports) [36]. Thus, although growth of cerebral cortical gray matter volume was prominent over the entire third trimester, a distinct change in rate was apparent over the second half of the third trimester, i.e., beginning at 33 to 34 weeks. Thus, cortical gray matter growth accelerated after 33-34 weeks, with its percentage of intracranial volume increasing from $29 \%$ prior to this inflection point to $47 \%$ after 33-34 weeks [36]. Cortical gray matter growth rate increased threefold after onset of the LPT period [36].

\section{Implications of rapid cerebral cortical development in the LPT infant}

A large clinical and experimental literature supports the general notion that preterm ex-utero exposure can lead to impaired brain maturation
$[14,37]$. In the case of the LPT infant, cerebral cortical maturation appears to be the most vulnerable target $[34,36]$. Moreover, overt injury to white matter does not appear to be an important factor, unlike the cortical dysmaturation observed in very preterm infants [14]. Experimental studies suggest that impaired cortical neuronal maturation (reduced dendritic arbor, dendritic retraction, decreased synaptic density) may occur after events as mild as transient hypoxemia [38] or perinatal stress [39]. A minority of LPT infants do have increased likelihood of such factors, as well as difficulties with resuscitation at birth, respiratory distress, hypothermia, hypoglycemia, hyperbilirubinemia and feeding difficulties [3]. Notably, however, as noted earlier, the majority of LPT infants have no clear neonatal morbidities and yet, still exhibit the neurocognitive deficits discussed.

\section{Conclusions}

Subsequent NDD affects a large absolute number of LPT infants (see earlier). Most of this disability affects infants without major neonatal morbidities. Clinical data suggest that this disability, largely categorized under the term, "cognitive deficits", involves functions of cerebral cortex. Recent imaging data suggest that the fundamental disturbance is impaired maturation of cerebral cortex, principally cerebral cortical neurons. Longstanding anatomic and recent imaging data indicate that cerebral cortical neuronal maturation is especially exuberant during the last weeks of gestation. Notably, one key study (see earlier) indicates that the period corresponding to the LPT infant is one of especially rapid developmental events involving cerebral cortex. Taken together, the findings suggest that the impaired maturation of cerebral cortex in the LPT infant is a new example of primary neuronal dysmaturation, previously identified by experimental and clinical studies in very and extremely preterm infants $[14,37]$.

The important question becomes how to prevent or ameliorate this cortical neuronal dysmaturation. This topic was addressed in previous discussions of very and extremely preterm infants $[14,37]$ and will not be reviewed in detail here. Notably with the LPT infant, prevention and treatment of such neonatal morbidities as hypoxemic events will be useful in the minority of infants so affected. Perhaps more importantly, in the total group of LPT infants, longer-term interventions involving nutrition, experiential factors (e.g., auditory, viscosity, pain and stress, parenting, education, 
and socio-economic factors, as described previously in detail in relation to very and extremely preterm infants $[14,37]$ will be critical in reducing the enormous burden of NDD in LPT infants.

\section{References}

[1] Hamilton BE, Martin JA, Osterman MJK. Births: Provisional Data for 2019. Vital Statistics Rapid Release, no-8. NCHS Data Brief 2020.

[2] Martinez-Nadal S, Bosch L. Cognitive and learning outcomes in late preterm infants at school age: A systematic review. Int J Environ Res Public Health. 2021;18:74.

[3] Huff K, Rose RS, Engle WA. Late preterm infants: Morbidities, mortality, and management recommendations. Pediatr Clin North Am. 2019;66:387-402.

[4] Vohr B. Long-term outcomes of moderately preterm, late preterm, and early term infants. Clin Perinatol. 2013;40: 739-51.

[5] Cheong JL, Doyle LW, Burnett AC, Lee KJ, Walsh JM, Potter CR, et al. Association between moderate and late preterm birth and neurodevelopment and social-emotional development at age 2 years. JAMA Pediatr. 2017;171:e164805.

[6] Heuvelman H, Abel K, Wicks S, Gardner R, Johnstone E, Lee B, et al. Gestational age at birth and risk of intellectual disability without a common genetic cause. Eur J Epidemiol. 2018;33:667-78.

[7] Woythaler M. Neurodevelopmental outcomes of the late preterm infant. Semin Fetal Neonatal Med. 2019;24:54-9.

[8] You J, Shamsi BH, Hao MC, Cao CH, Yang WY. A study on the neurodevelopment outcomes of late preterm infants. BMC Neurol. 2019;19:108.

[9] Favrais G, Saliba E. Neurodevelopmental outcome of latepreterm infants: Literature review. Arch Pediatr. 2019;26: 492-6.

[10] Martinez-Nadal S, Demestre X, Schonhaut L, Munoz SR, Sala P. Impact of neonatal morbidity on the risk of developmental delay in late preterm infants. Early Hum Dev. 2018; 116:40-6.

[11] Petrini JR, Dias T, McCormick MC, Massolo ML, Green NS, Escobar GJ. Increased risk of adverse neurological development for late preterm infants. J Pediatr. 2009;154: 169-76.

[12] Hirvonen M, Ojala R, Korhonen P, Haataja P, Eriksson K, Gissler M, et al. Cerebral palsy among children born moderately and late preterm. Pediatrics. 2014;134:e1584-93.

[13] Haynes RL, Sleeper LA, Volpe JJ, Kinney HC. Neuropathologic studies of the encephalopathy of prematurity in the late preterm infant. Clin Perinatol. 2013;40:707-22.

[14] Volpe JJ. Dysmaturation of premature brain: Importance, cellular mechanisms and potential interventions. Pediatr Neurol. 2019;95:42-66.

[15] Back SA, Luo NL, Borenstein NS, Levine JM, Volpe JJ, Kinney HC. Late oligodendrocyte progenitors coincide with the developmental window of vulnerability for human perinatal white matter injury. J Neurosci. 2001;21:1302-12.

[16] Degnan AJ, Wisnowski JL, Choi S, Ceschin R, Bhushan C, Leahy RM, et al. Alterations of resting state networks and structural connectivity in relation to the prefrontal and anterior cingulate cortices in late prematurity. Neuroreport. 2015;26:22-6.
[17] Kelly CE, Cheong JL, Gabra Fam L, Leemans A, Seal ML, Doyle LW, et al. Moderate and late preterm infants exhibit widespread brain white matter microstructure alterations at term-equivalent age relative to term-born controls. Brain Imaging Behav. 2016;10:41-9.

[18] Munakata S, Okada T, Okahashi A, Yoshikawa K, Usukura Y, Makimoto M, et al. Gray matter volumetric MRI differences late-preterm and term infants. Brain Dev. 2013;35: $10-6$.

[19] Walsh JM, Doyle LW, Anderson PJ, Lee KJ, Cheong JL. Moderate and late preterm birth: effect on brain size and maturation at term-equivalent age. Radiology. 2014;273:232-40.

[20] Kim DJ, Davis EP, Sandman CA, Sporns O, O'Donnell BF, Buss $\mathrm{C}$, et al. Longer gestation is associated with more efficient brain networks in preadolescent children. Neuroimage. 2014;100:619-27.

[21] Rogers CE, Barch DM, Sylvester CM, Pagliaccio D, Harms MP, Botteron KN, et al. Altered gray matter volume and school age anxiety in children born late preterm. J Pediatr. 2014;165:928-35.

[22] Wu X, Wei L, Wang N, Hu Z, Wang L, Ma J, et al. Frequency of spontaneous BOLD signal differences between moderate and late preterm newborns and term newborns. Neurotox Res. 2016;30:539-51.

[23] Cheong JL, Thompson DK, Spittle AJ, Potter CR, Walsh $\mathrm{JM}$, Burnett AC, et al. Brain volumes at term-equivalent age are associated with 2-year neurodevelopment in moderate and late preterm children. J Pediatr. 2016;174g:91-7 e1.

[24] Brumbaugh JE, Conrad AL, Lee JK, DeVolder IJ, Zimmerman MB, Magnotta VA, et al. Altered brain function, structure, and developmental trajectory in children born late preterm. Pediatr Res. 2016;80:197-203.

[25] Batalle D, Hughes EJ, Zhang H, Tournier JD, Tusor N, Aljabar P, et al. Early development of structural networks and the impact of prematurity on brain connectivity. Neuroimage. 2017;149:379-92.

[26] Cheong JLY, Thompson DK, Olsen JE, Spittle AJ. Late preterm births: New insights from neonatal neuroimaging and neurobehaviour. Semin Fetal Neonatal Med. 2019; 24:60-5.

[27] Williams LZJ, McNamara D, Alsweiler JM. Intermittent hypoxemia in infants born late preterm: A prospective cohort observational study. J Pediatr. 2019;204: 89-95 e1.

[28] Marin-Padilla M. Ontogenesis of the pyramidal cell of the mammalian neocortex and developmental cytoarchitectonics: A unifying theory. J Comp Neurol. 1992;321: 233-40.

[29] Bouyssi-Kobar M, du Plessis AJ, McCarter R, BrossardRacine M, Murnick J, Tinkleman L, et al. Third trimester brain growth in preterm infants compared with in utero healthy fetuses. Pediatrics. 2016;138.

[30] Andescavage NN, du Plessis A, McCarter R, Serag A, Evangelou I, Vezina $G$, et al. Complex trajectories of brain development in the healthy human fetus. Cereb Cortex. 2017;27:5274-83.

[31] Huppi PS, Warfield S, Kikinis R, Barnes PD, Zientara GP, Jolesz FA, et al. Quantitative magnetic resonance imaging of brain development in premature and mature newborns. Ann Neurol. 1998;43:224-35.

[32] Kapellou O, Counsell SJ, Kennea NL, Dyet L, Saeed N, Stark J, et al. Abnormal cortical development after premature birth shown by altered allometric scaling of brain growth. PLOS Medicine. 2006;3:e265. 
[33] Rathbone R, Counsell SJ, Kapellou O, Dyet L, Kennea N, Hajnal J, et al. Perinatal cortical growth and childhood neurocognitive abilities. Neurology. 2011;77:1510-7.

[34] Ball G, Latha S, Aljabar P, Counsell S, Durighel G, Hajnal J, et al. Development of cortical microstructure in the preterm human brain. Proc Natl Acad Sci (USA). 2013;110:9541-6.

[35] Moeskops P, Benders MJ, Kersbergen KJ, Groenendaal F, de Vries LS, Viergever MA, et al. Development of cortical morphology evaluated with longitudinal MR brain images of preterm infants. PLoS One. 2015;10:e0131552.

[36] Matthews LG, Walsh BH, Knutsen C, Neil JJ, Smyser CD, Rogers CE, et al. Brain growth in the NICU: Critical periods of tissue-specific expansion. Pediatr Res. 2018;83:976-81.
[37] Volpe JJ. Primary neuronal dysmaturation in preterm brain: Important and likely modifiable. J Neonatal Perinatal Med. 2021:1-6.

[38] Dean JM, McClendon E, Hansen K, Azimi-Zonooz A, Chen K, Riddle A, et al. Prenatal cerebral ischemia disrupts MRI-defined cortical microstructure through disturbances in neuronal arborization. Sci Transl Med. 2013;5:168ra7.

[39] Murmu MS, Salomon S, Biala Y, Weinstock M, Braun K, Bock J. Changes of spine density and dendritic complexity in the prefrontal cortex in offspring of mothers exposed to stress during pregnancy. Eur J Neurosci. 2006;24:1477-87. 